\title{
Pim-1 Kinase Cooperates with Serum Signals Supporting Mesenchymal Stem Cell Propagation
}

\author{
Yunshan Zhao ${ }^{a}$ Jingping Hu ${ }^{a}$ Bryan Buckingham ${ }^{a}$ Mark F. Pittenger ${ }^{a}$ \\ Zhongjun J. Wu ${ }^{a}$ Bartley P. Griffith ${ }^{b}$ \\ ${ }^{a}$ Artificial Organ Lab, University of Maryland School of Medicine, and ${ }^{b}$ Department of Surgery, University of \\ Maryland, Baltimore, Md., USA
}

\section{Key Words}

Pim-1 kinase - Mesenchymal stem cell propagation .

Cell regulation - Growth and survival · Ovine model

\begin{abstract}
Mesenchymal stem cells (MSCs) are currently undergoing testing in several clinical settings. The propagation of MSCs from multiple species in culture is an important step in furthering our understanding of these progenitor cells. Pim-1, a proto-oncogenic serine/threonine kinase, regulates cell proliferation, survival, and differentiation. Although it has been shown that Pim-1 participates in signal transduction mediating mitogenic action in MSCs, its roles in the modulation of MSC propagation remain to be defined. Understanding of ovine MSCs transduced with Pim-1 may provide improved ovine models for cellular therapy development. Using genetically modified ovine MSCs that constitutively overexpressed Pim-1 (MSC expressing PIM-1 and ZsGreen protein), we evaluated the impact of elevated Pim- 1 activity on the proliferation, survival, and differentiation of MSCs in culture. Our results showed that Pim-1 enhanced the intrinsic molecular signals of growth and survival implicated in the mediation of serum signaling under normal culture conditions ( $10 \%$ serum). We found that Pim-1 promoted MSC pro-
\end{abstract}

\section{KARGER}

(c) 2014 S. Karger AG, Basel

$1422-6405 / 14 / 1993-0140 \$ 39.50 / 0$

E-Mail karger@karger.com

www.karger.com/cto liferation irrespectively of the serum concentration, but with a decreased proliferation rate compared to increased serum concentrations, relative to the control vector-transduced MSC expressing ZsGreen protein. Further, Pim-1 prevented MSC apoptosis induced by hypoxia or serum deprivation as evidenced by enhanced mitochondria integrity and reduced annexin $\mathrm{V}$ binding. Interestingly, the phenotype and multilineage differentiation potential of the cells were not influenced by Pim-1. Taken together, these observations demonstrate that Pim-1 kinase cooperates with exogenous serum signals supporting MSC propagation in the ovine model.

๑) 2014 S. Karger AG, Basel

\section{Introduction}

Modifications of molecular signaling networks during development, and protection against pathological insults and tissue regeneration are finely regulated by kinases during cell and tissue growth and injury survival. Pim-1, a proto-oncogenic serine/threonine kinase, can be acti-

Prof. Bartley P. Griffith, MD

Department of Surgery, University of Maryland, Baltimore N4W94, 22 S Greene St, Baltimore, MD 21201-1116 (USA)

E-Mail bgriffith@smail.umaryland.edu

Prof. Zhongjun J. Wu, PhD

Artificial Organ Lab, University of Maryland School of Medicine

10 South Pine Street, Room 443

Baltimore, MD 21201-1116 (USA)

E-Mail zwu@smail.umaryland.edu 


\begin{tabular}{ll}
\hline Abbreviations used in this paper \\
\hline Bcl-2 & B-cell lymphoma 2 \\
Bcl-xl & B-cell lymphoma-extra large \\
cdk2 & cyclin-dependent kinase 2 \\
FBS & fetal bovine serum \\
GAPDH & glyceraldehyde-3-phosphate dehydrogenase \\
MSCs & mesenchymal stem cells \\
MSCz & mesenchymal stem cells expressing ZsGreen \\
& protein \\
MSCzP & mesenchymal stem cells expressing Pim-1 and \\
& ZsGreen proteins \\
MTT & 3-4, 5-dimethylthiazol-2,5-diphenyltetrazolium \\
SD & serum deprivation \\
TMRE & tetramethylrhodamine ethyl ester \\
$\Delta \Psi m$ & mitochondrial inner membrane potential \\
\hline
\end{tabular}

vated by growth factors, cytokines, and mechanical stimulation and has been demonstrated to be involved in the regulation of signaling networks related to cell proliferation, apoptosis, and differentiation [Wang et al., 2001; Aho et al., 2004; Bachmann and Moroy, 2005]. Pim-1 expression decreases during postnatal development but reemerges following acute pathological injury and is increased in failing hearts, exerting protective actions on cardiomyocytes from apoptosis and stimulating cardiac progenitor cell cycling in the infarcted heart [Muraski, et al., 2007; Borillo et al., 2010; Cottage et al., 2010]. Pim-1 expression has been observed in balloon-injured rat carotid arteries, and dominant negative Pim-1 markedly suppressed the proliferation of vascular smooth muscle cells in the balloon-injury model [Katakami et al., 2004]. In murine embryonic stem cells, the Pim-1 level is elevated by leukemia inhibitory factor/gp130-dependent signaling and the STAT3 transcription factor and, as such, enhances the ability of murine embryonic stem cell selfrenewal and the resistance to leukemia inhibitory factor starvation [Aksoy et al., 2007]. Additionally, Pim-1 is upregulated by STAT3 activation in cardiac Sca-1+ progenitor cells and plays a pivotal role in endothelial cell differentiation both in vitro and in vivo [Iwakura et al., 2011]. Thus, Pim-1 mediates proliferative signaling and protective effects and critically regulates cell growth, survival, and differentiation when naturally activated in normal cells.

Mesenchymal stem cells (MSCs) are a stem cell population present in most adult tissues, and their ability to give rise to a variety of cell types and release paracrine soluble factors, together with their extensive capacity for in vitro expansion, makes them one of the most attractive populations of stem cells for tissue engineering and cell therapy [Pittenger and Martin, 2004; Gnecchi et al., 2008; Spiropoulos et al., 2011; Williams and Hare, 2011]. Propagation of MSCs in the fetal bovine serum (FBS) culture system is thus far the most successful strategy for MSC expansion. The signals of growth factors and cytokines that serum provides are mediated via transduction pathways such as the ERK/MEK/Raf pathway and target downstream substrates related to regulation of the survival machinery and the cell cycle, initiating MSC proliferation and maintaining cell growth and survival as well as the cell phenotype and multilineage differentiation potential [Gineitis and Treisman, 2001; Pittenger and Martin, 2004; Spiropoulos et al., 2011]. Usually, MSCs are expanded under normal culture conditions containing $10 \%$ FBS, and low serum results in decreased proliferation while deprivation of serum induces MSC apoptosis. Recently, Tu et al. [2011] reported the activation of Pim-1 by shear stress which exerted mitogenic action resulting in an increased BrdU uptake under normal culture conditions in MSCs. This suggests an important modulation of Pim-1 on MSC proliferation through interaction with serum signals, but the roles of Pim-1 in the regulation of MSC propagation in a serum culture system remain to be defined. Using genetically modified MSCs that constitutively expressed Pim-1, the impact of elevated Pim-1 activity on MSC growth, survival, and differentiation was examined at the molecular and cellular levels. Results demonstrated that Pim-1 enhanced intrinsic molecular signals of survival and growth implicated in the mediation of serum signals, promoted and maintained MSC proliferation in a serum culture system, and reduced apoptosis induced by serum deprivation (SD), but maintained the phenotype and multilineage differentiation potential. These findings demonstrate that Pim-1-mediated signaling cooperates with exogenous serum signals supporting MSC propagation.

\section{Materials and Methods}

Construction of a Lentiviral Vector Expressing Pim-1

RNA was extracted from ovine cardiac tissue using an RNeasy mini kit, and reverse transcription was carried out with the SuperScript III First-Stand Synthesis System (Life Technologies, Grand Island, N.Y., USA). The resulting cDNA was amplified from the primers of $5^{\prime}$-GAA TTC ATG CTC TTG TCC AAA ATC AAC- $3^{\prime}$ and $5^{\prime}$-TCG CCA GGG CCC AGC AAA TAG CGG CCG-3'. The restriction sites of EcoRI and Not I are underlined. The PCR product was cloned and transformed using a TA cloning kit, and then the purified $5^{\prime}$-EcoRI/NotI- $3^{\prime}$ fragment containing the entire Pim- 
1 gene was subcloned into a pLVX-IRES-ZsGreen 1 lentiviral vector (Clontech, Mountain View, Calif., USA) for the construction of plasmid pLVX-IRES-ZsGreen1-Pim-1.

\section{Lentivirus Production}

Lentivirus expressing Pim-1 was prepared using the Lenti-X HT Packaging System (Clontech) according to the manufacturer's protocol. Briefly, $5 \times 10^{6} \mathrm{HEK} 293$ cells were seeded in a $100-\mathrm{mm}$ dish containing tetracycline-free $10 \%$ FBS MEM growth medium. The cells were transfected the next day with pLVX-IRES-ZsGreen1 or recombinant pLVX-IRES-ZsGreen1-Pim-1 vector DNA together with the Lenti-X HT Packaging Mix and Lentiphos 1 and 2. After overnight incubation, the transfection medium was replaced with $10 \mathrm{ml}$ fresh tetracycline-free 10\% FBS MEM growth medium. Tet-Off transactivation is used to drive the high-level expression of specific viral packaging proteins. The lentivirus-containing supernatants were harvested after $48 \mathrm{~h}$ of incubation and stored frozen.

\section{Isolation and Culture of MSCs}

MSCs were isolated from bone marrow using our previously published protocol [Zhao et al., 2012]. Briefly, fresh bone marrow aspirates were obtained from iliac crests of adult sheep (approximately $1-4$ years old, $n=3$ ) under general anesthesia according to procedures approved by the Institutional Animal Care and Use Committee of the University of Maryland School of Medicine. The bone marrow aspirates were diluted with $0.9 \% \mathrm{NaCl}$ and filtered $(70 \mu \mathrm{m})$. The mononuclear cells were isolated by density gradient centrifugation using Ficoll-Paque Premium $(1.077 \mathrm{~g} / \mathrm{ml}$; GE Healthcare, Uppsala, Sweden) at 1,400 rpm for $40 \mathrm{~min}$ at room temperature. The mononuclear cells were washed with PBS and incubated in low-glucose Dulbecco's Modified Eagle Medium (Life Technologies, Grand Island, N.Y., USA) containing 15\% MSC-qualified FBS (Hyclone). After $24 \mathrm{~h}$ of incubation, nonadherent cells were removed and adherent cells were subcultured using TrypLE Express (Life Technologies) when the cells achieved $70-80 \%$ confluence.

\section{Transduction of MSCs with Lentivirus}

Ovine MSCs from passage 1 were cultured in complete growth medium overnight. Transduction of MSCs was performed by incubating the lentiviral stocks of either pLVX-IRES-ZsGreen-1 or pLVX-IRES-ZsGreen1-Pim-1 with the cells for $8 \mathrm{~h}$ in the presence of $4 \mu \mathrm{g} / \mathrm{ml}$ polybrene. Thereafter, transduction media were removed and replaced with fresh culture medium. After $48 \mathrm{~h}$ of transduction, the transduction efficiency was analyzed by flow cytometry based on lentiviruses expressing ZsGreen. The lentivirusinfected populations were further sorted via fluorescence-activated cell sorting, expanded, and used in the experiments.

\section{Western Blot}

Immunoblotting was performed as described previously [Yankey et al., 2008]. In brief, cell samples from cultured MSCs, MSCs expressing ZsGreen protein (MSCz), and MSCs expressing Pim-1 and ZsGreen proteins (MSCzP) were homogenized in immunoprecipitation assay buffer, separated by SDS-polyacrylamide gel electrophoresis, transferred to a polyvinylidene difluoride membrane, and probed with specific primary antibodies. Protein loading was controlled by normalization to the expression of glyceraldehyde-3-phosphate dehydrogenase (GAPDH; Santa Cruz Bio- technology Inc., Santa Cruz, Calif., USA). Western blots for the expression of Pim-1, extracellular signal-regulated kinases (ERK and phosphorylated p-ERK), phosphorylated Raf (Cell Signaling Technology, Danvers, Mass., USA), B-cell lymphoma-extra large (Bcl-xl; Cell Signaling Technology), B-cell lymphoma 2 (Bcl-2; Cell Signaling Technology), p27 Kip1 and phosphorylated p2 $7^{\text {Kip } 1}$ (Cell Signaling Technology), and c-myc (Cell Signaling Technology) were digitized and quantified with UN-SCAN-IT gel TM 5.1 software (Silk Scientific, Orem, Utah, USA) using enhanced chemiluminescence (Amersham Biosciences, Piscataway, N.J., USA). Densitometric results were expressed as the ratio of the target protein to GAPDH.

\section{Cell Growth Curve}

MSCs, MSCz, or MSCzP (passage 4) were seeded onto 96-well plates at a density of $1 \times 10^{3} /$ well and cultured in medium containing 10,5 , or $1 \%$ FBS (Gibco). The medium was changed every 2 days via a $50 \%$ medium change. Cell numbers were determined using a 3-4, 5-dimethylthiazol-2,5-diphenyltetrazolium (MTT) assay according to the manufacturer's instructions (Roche, Indianapolis, Ind., USA) every day for 7 days. The data represented an average of 3 duplicates, and growth curves were plotted. The relative proliferation rate was calculated as $\left(\mathrm{OD}_{\mathrm{MSCz}}-\mathrm{OD}_{\mathrm{MSCz}}\right) / \mathrm{OD}_{\mathrm{MSCz}}$.

\section{Tetramethylrhodamine Ethyl Ester Assay}

Tetramethylrhodamine ethyl ester (TMRE) assay was used to detect mitochondria membrane depolarization of MSCs based on the manufacturer's protocol (MitoPT ${ }^{\mathrm{TM}}$; ImmunoChemistry Technologies, Bloomington, Minn., USA). Briefly, cells (passage 4) were trypsinized and resuspended in staining buffer. Thereafter, TMRE (20-200 nM) solution was added to the cell suspension, followed by incubation for $15 \mathrm{~min}$ at $37^{\circ} \mathrm{C}$ and $5 \% \mathrm{CO}_{2}$ in the dark. The stained cells were washed once with wash buffer and resuspended in PBS. The cells were then challenged with $100 \mu \mathrm{M} \mathrm{H}_{2} \mathrm{O}_{2}$, and fluorescence was immediately analyzed using a plate reader.

\section{Flow Cytometry}

Flow cytometric analysis was used to characterize cell surface antigen expression using markers positively and negatively associated with MSC populations derived from other species and to determine cell apoptosis based on annexin V binding. Briefly, MSCs, $\mathrm{MSC} z$, or MSCzP (each at passage 3) were incubated with primary antibody mouse anti-sheep CD44 (AbD Serotec; Raleigh, N.C., USA; http://www.ab-direct.com), mouse anti-human CD166 (BD Biosciences, San Diego, Calif., USA; http://www.bdbiosciences. com), mouse anti-human CD34 (BD Biosciences), mouse antisheep CD45 (AbD Serotec), or isotype-matched control (AbD Serotec) for $20 \mathrm{~min}$ at room temperature. After washing to remove unbound primary antibody, cells were incubated for $20 \mathrm{~min}$ in a 1: 100 dilution of secondary Alexa Fluor 488-conjugated goat antimouse IgG on ice. For the annexin V binding apoptosis assay, cells were cultured in media without serum and placed in a hypoxia incubator chamber (STEMCELL Technologies Inc., Vancouver, B.C., Canada) for $24 \mathrm{~h}$, and then annexin $\mathrm{V}$ binding was performed and assessed according to the manufacturer's instructions. Flow cytometric analysis was performed using an Epics1-XL-MCL analyzer (Beckman Coulter, Fullerton, Calif., USA). Typically, for each sample, 10,000 events were collected on the flow cytometer and stored as list mode data. Off-line analysis was performed using WinMDI software (Windows Multiple Document Interface Flow Cytometry 
Fig. 1. Regulation of key proteins implicated in the mediation of serum signaling by Pim-1. a Representative photomicrographs of Western blots showing upregulation of phosphorylated ERK, phosphorylated Raf, Bcl-xl, p27 ${ }^{\mathrm{Kip} 1}$, and phosphorylated p27 ${ }^{\mathrm{Kip} 1}$, and the unaltered expression of c-myc and ERK as well as downregulation of $\mathrm{Bcl}-2$ in MSCzP compared to the controls of MSCs or MSCz along with Pim-1 overexpression. b Quantitative graphs representing the relative levels of ERK, phosphorylated ERK, phosphorylated Raf, p27 $7^{\mathrm{Kip} 1}$, and phosphorylated $\mathrm{p} 27^{\mathrm{Kip} 1}$ normalized to GAPDH expressed in MSCz and MSCzP $(n=3)$.

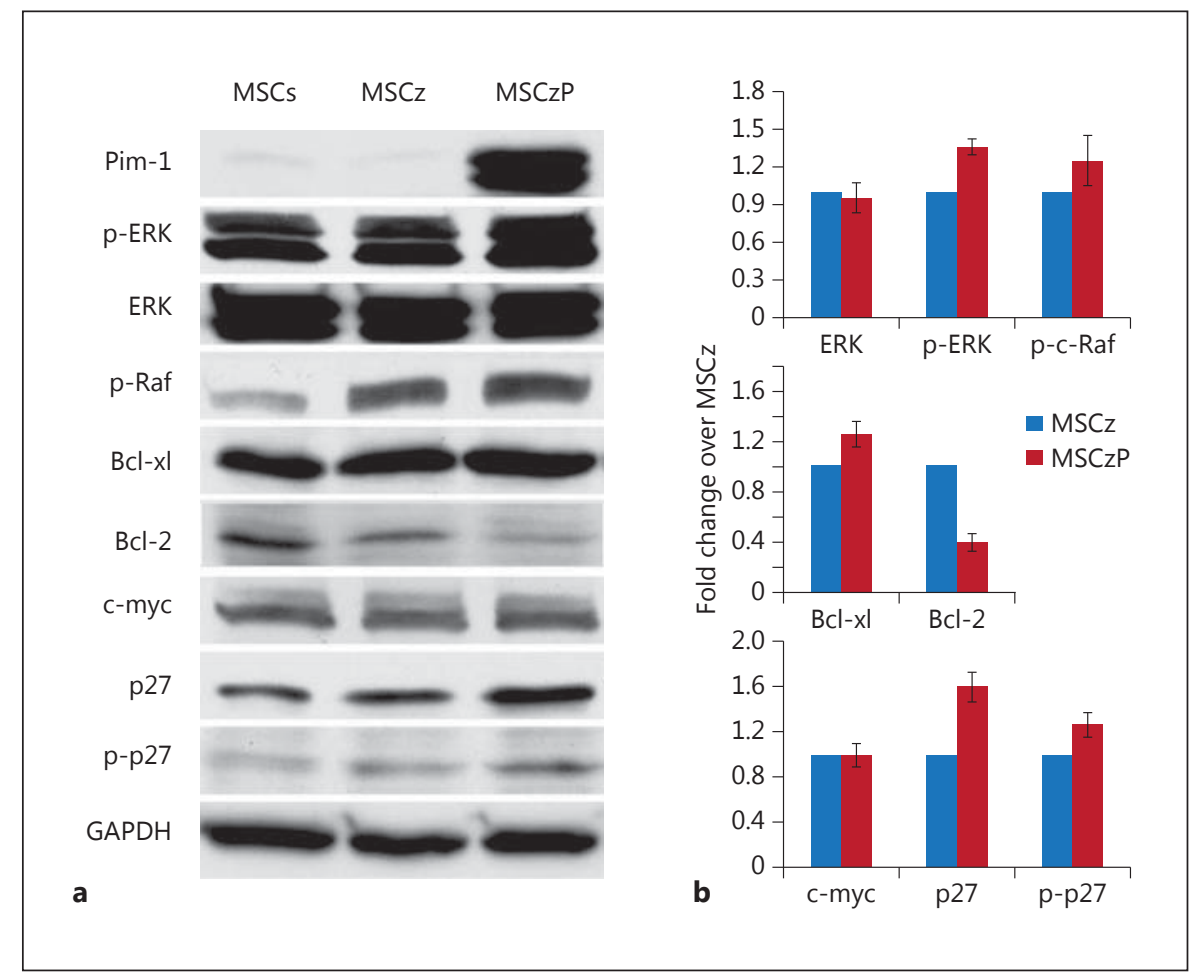

Application; Joseph Trotter, copyright 1993-1998). Positive fluorescence was defined as a level of fluorescence $>99 \%$ of the corresponding isotype-matched control antibody.

\section{Trilineage Differentiation}

Multipotential differentiation of the ovine MSCs, MSCz, and MSCzP towards osteoblasts, chondrocytes, and adipocytes was evaluated based on a protocol described previously [Zhao et al., 2012 ]. Briefly, cells at passage 4 were seeded onto 12 -well plates at $1 \times 10^{4}$ cells $/ \mathrm{cm}^{2}$ for adipogenesis and at $5 \times 10^{3} \mathrm{cells} / \mathrm{cm}^{2}$ for osteogenesis in Dulbecco's modified Eagle's medium supplemented with penicillin $(100 \mathrm{IU} / \mathrm{ml}) /$ streptomycin $(100 \mu \mathrm{g} / \mathrm{ml})$ and $10 \%$ (volume/volume) MSC FBS (Gibco) and incubated at $37^{\circ} \mathrm{C}$ in the presence of $5 \% \mathrm{CO}_{2}$. For differentiation of MSCs towards chondrogenesis, micromasses were generated by plating 5 - $\mu$ l droplets of cell solution of $1.6 \times 10^{7} / \mathrm{ml}$ in the center of multiwell plate wells under the above described culture conditions. Two days later, the culture medium was replaced with differentiation medium (Gibco) and changed every 2 days thereafter. At 14 days for adipogenesis and 28 days for osteogenesis and chondrogenesis, samples were fixed with $4 \%$ paraformaldehyde for $30 \mathrm{~min}$. Histological staining was performed using Oil Red $\mathrm{O}$ for adipocytes, Alcian Blue for chondrocytes, and alizarin red for osteoblasts.

\section{Statistical Analysis}

Data are presented as means \pm SEM. A post hoc analysis, along with one-way repeated-measures ANOVA, was used to compare differences among groups. All ANOVAs were followed by multiple comparisons with the least-significant-difference correction. $\mathrm{p}<0.05$ was considered statistically significant.

Pim-1 Kinase Supports MSC Propagation

\section{Results}

\section{Pim-1 Enhanced Intrinsic Molecular Signals of Growth and Survival in MSCs}

Based on previous reports that Pim-1 regulated the expression of signaling molecules related to growth and survival [Aho et al., 2004; Muraski et al., 2007; Borillo et al., 2010], we first examined the levels of key proteins implicated in these processes to assess the effects of elevated Pim-1 activity on intrinsic molecular signals in MSCs. The level of phosphorylated ERK had a 1.4-fold increase along with Pim-1 overexpression in MSCzP compared to the control of MSCz in which Pim-1 expression was undetectable, although the levels of ERK were comparable in MSCz and MSCzP (fig. 1a, b). Similarly, the level of phosphorylated Raf had a 1.2-fold increase in MSCzP relative to that in $\mathrm{MSCz}$ (fig. 1a, b). Interestingly, the expression of the Bcl-2 family proteins $\mathrm{Bcl}-\mathrm{xl}$ and $\mathrm{Bcl}-2$, which are regulators of the mitochondria-mediated apoptosis pathway, was differentially regulated by Pim-1. The expression of the antiapoptotic protein Bcl-xl had a 1.2-fold increase in MSCzP, while the expression of the proapoptotic protein $\mathrm{Bcl}-2$ decreased 0.4-fold compared to the control of $\mathrm{MSCz}$ (fig. 1a, b). In contrast, the level of c-Myc remained similar in $\mathrm{MSCz}$ and $\mathrm{MSCzP}$, while 

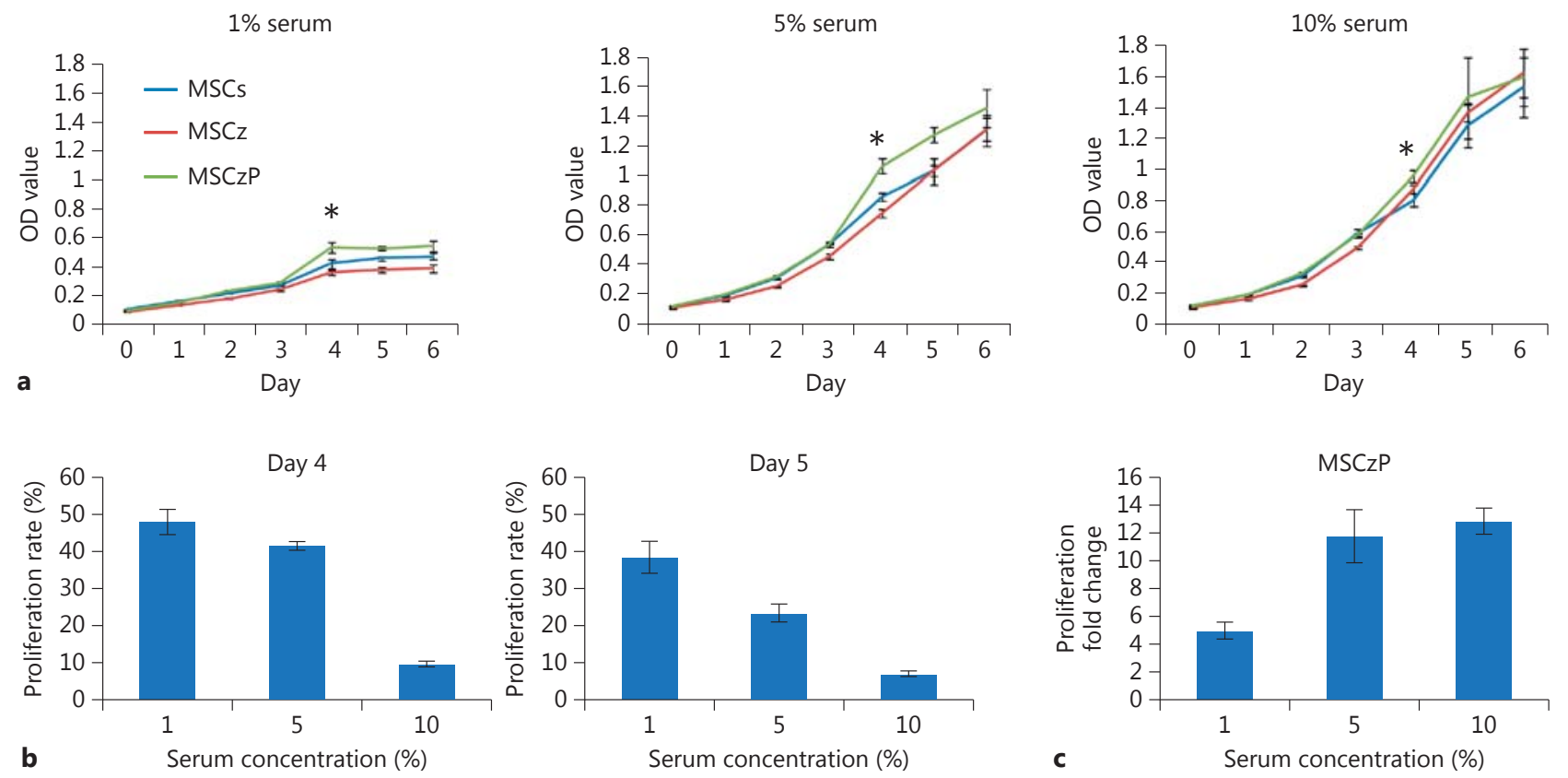

Fig. 2. Effects of Pim-1 on MSC proliferation in media containing different concentrations of serum. Growth curve of MSCs, MSCz, and MSCzP in media containing 1,5, and $10 \%$ serum showing that Pim-1 promoted MSC proliferation irrespectively of the serum concentration. The asterisk indicates the significant increase in cell proliferation (MSCzP vs. MSCs or MSCz) on day 4. $(n=3)$. b Relative proliferation rates of MSCzP compared to MSCz in media containing 1,5 , and $10 \%$ serum showing decreased proliferation rates over increased serum concentrations $(n=3)$. c Proliferation of MSCzP compared to the initial cell number during a 6 -day culture showing the comparable increase in MSCzP in proliferation at $5 \%$ serum compared to that at $10 \%$. $(n=3)$.

(fig. 2a). Compared to the control MSCs or MSCz, MSCzP proliferated faster during the exponential phase of the fourth day after plating until the plateau phase, irrespectively of the serum concentration used in the medium (fig. 2a). However, the relative proliferation rates of MSCzP compared to MSCz varied with the serum concentration and decreased with an increase in serum concentration in the medium; it was highest at $1 \%$ serum, secondary at 5\%, and lowest at $10 \%$ (fig. 2b). Moreover, MSCzP showed an 11.8-fold increase in proliferation at $5 \%$ serum comparable to the 12.8 -fold increase at $10 \%$ serum relative to the initial cell number during the 7 -day culture (fig. 2c); in contrast, $\mathrm{MSCzP}$ rapidly reached the plateau phase and only had a 4.9 -fold increase at $1 \%$ serum, showing the dependence of Pim-1 in regulating ovine MSC proliferation on the serum concentration (fig. 2c). typical sigmoidally shaped growth curve, with a slow start, an exponential phase, and then a plateau phase 


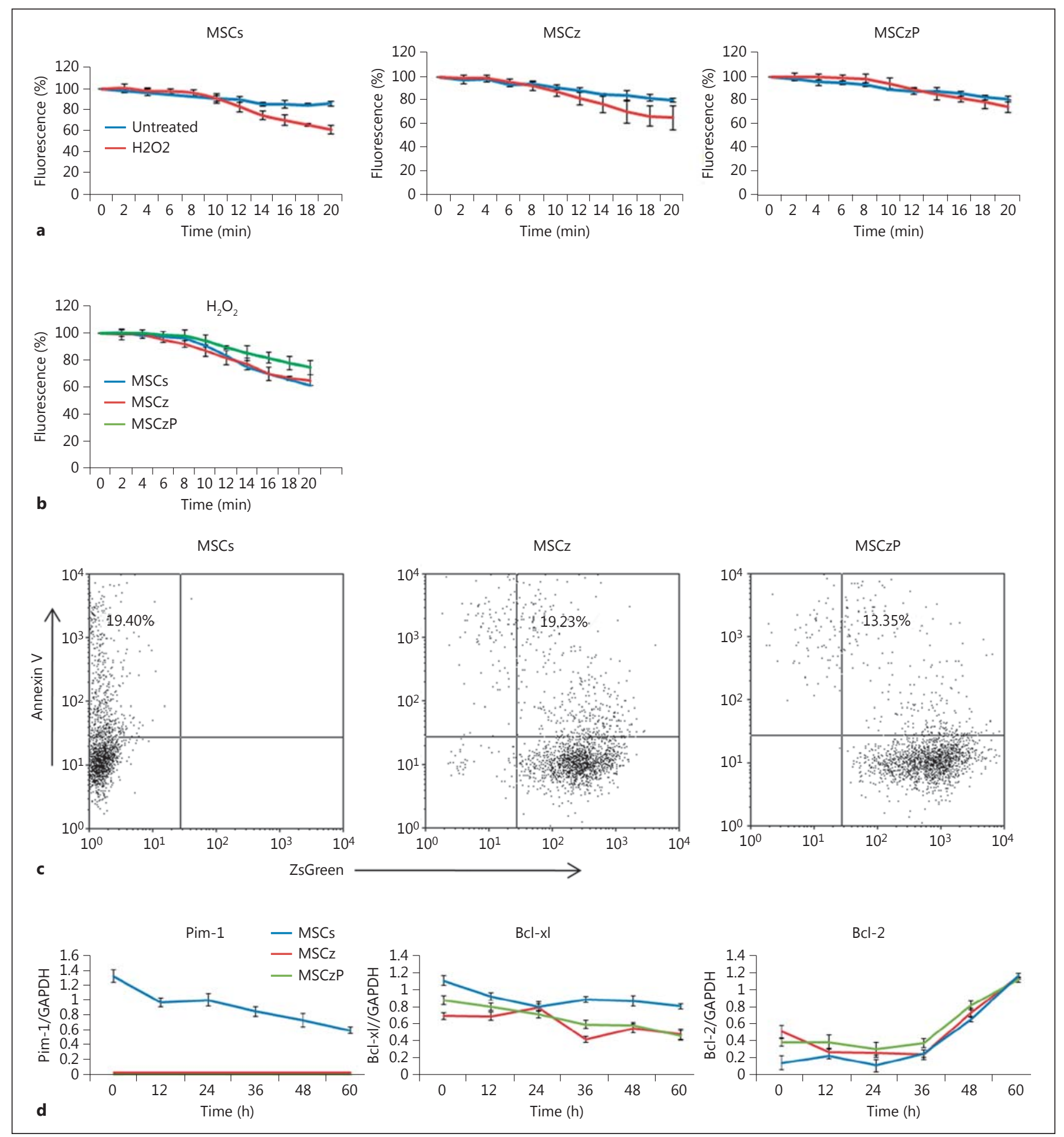

Fig. 3. Effects of hypoxia/SD-induced MSC apoptosis by Pim-1. a-c Mitochondrial inner membrane depolarization in MSCs induced by $\mathrm{H}_{2} \mathrm{O}_{2}$ is delayed by Pim-1 overexpression. TMRE fluorescence of MSCs, MSCz, and $\mathrm{MSCzP}$ under control conditions (untreated) vs. an oxidative stress challenge $\left(\mathrm{H}_{2} \mathrm{O}_{2} ; 100 \mu \mathrm{mol} / \mathrm{l} ; \mathbf{a}\right)$; combined plot comparing the results of $\mathbf{a}(\mathbf{b} ; \mathrm{n}=3)$. c Flow cytometric analysis of cell apoptosis $24 \mathrm{~h}$ after exposure to hypoxia/SD based on the binding of annexin V in MSCs, MSCz, and MSCzP demonstrating the inhibition of Pim-1 on MSC apoptosis induced by hypoxia/SD $(\mathrm{n}=3)$. d Expression kinetics of Pim-1, Bcl-xl, and Bcl-2 proteins using Western blot during $48 \mathrm{~h}$ of treatment with hypoxia/SD in MSCs, MSCz, and MSCzP showing that the expression of Pim-1, Bcl-xl, and Bcl-2 decreased with the duration of hypoxia/SD $(\mathrm{n}=3)$. 
Fig. 4. Effects of Pim-1 kinase on the MSC phenotype and differentiation potential. a Flow cytometry analysis of cell surface markers demonstrating that MSCzP maintained the expression of cell surface markers $(\mathrm{n}=3)$. $\mathbf{b}$ Cell morphology of MSCs, MSCz, and $\mathrm{MSCz}$ in culture showing that MSCzP retained the typical morphology of MSCs, with a spindle-shaped, fibroblastic morphology similar to that of MSCs and MSCz. c Multipotential differentiation towards adipogenesis, osteogenesis, and chondrogenesis. Osteogenesis differentiation showed calcium nodules stained with alizarin red. Chondrogenesis differentiation showed the matrix stained with alcian blue. Adipogenesis differentiation showed the adipocyte droplets stained with Oil Red O.

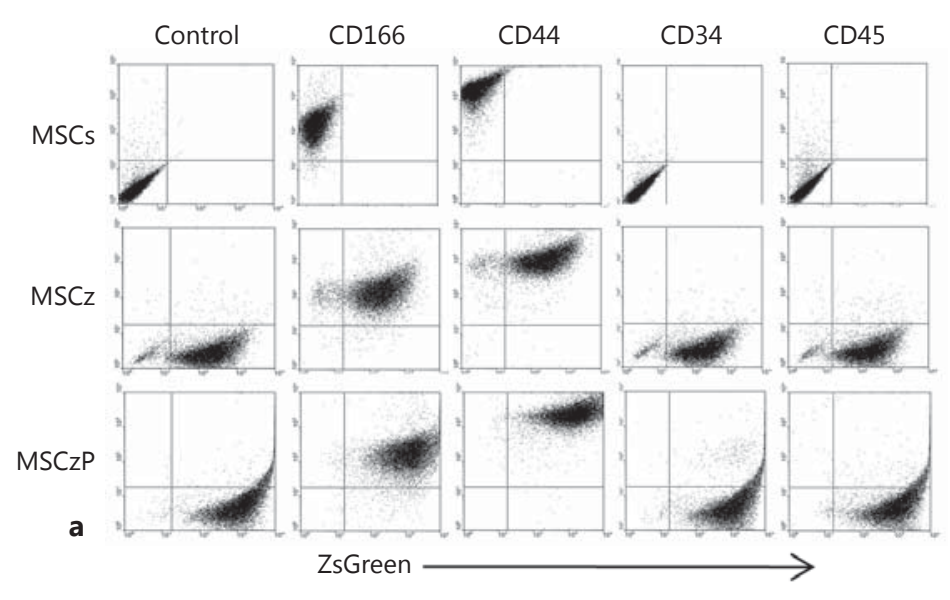

b
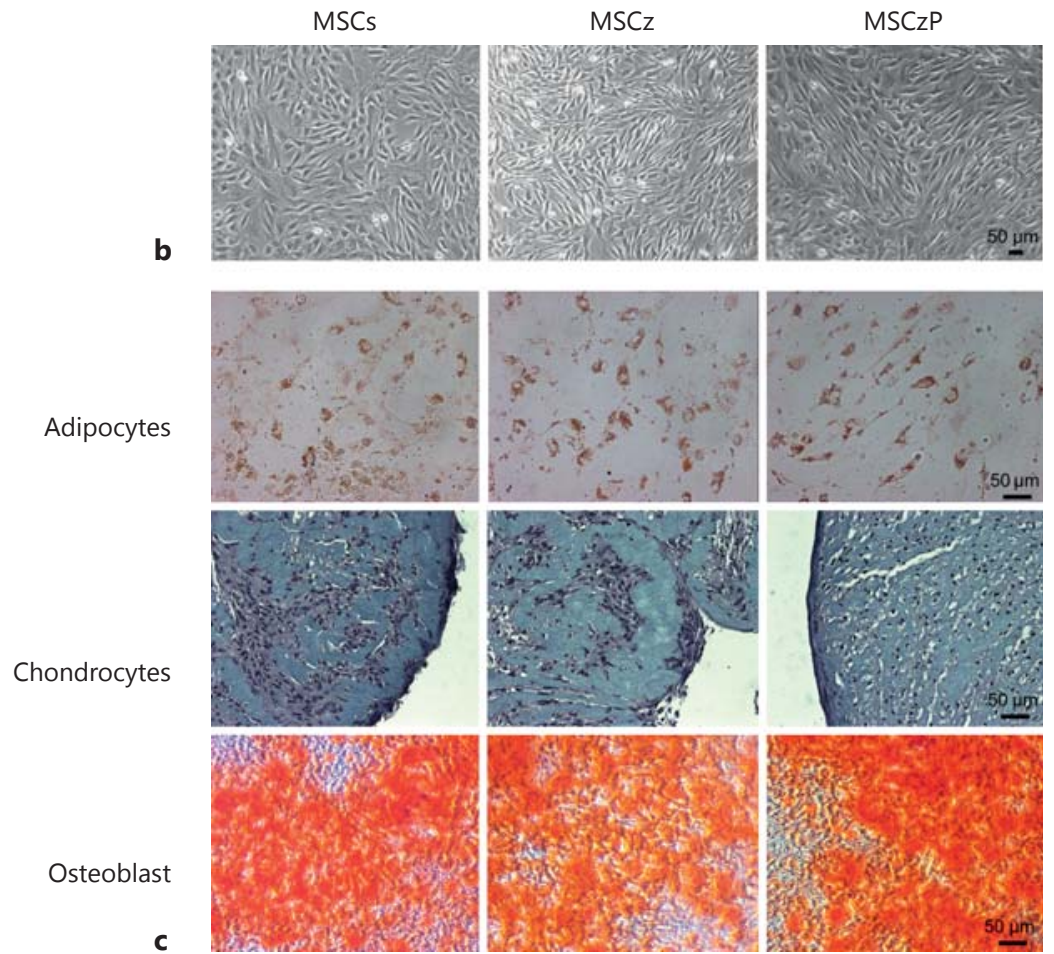

Pim-1 Showed Inhibition of MSC Apoptosis Induced by Hypoxia and SD

Accumulating evidence has shown that MSC apoptosis induced by hypoxia or SD occurs mainly through a mitochondria-mediated pathway [Zhu et al., 2006; Deng et al., 2010]. We therefore examined the effects of elevated Pim-1 activity on the preservation of the mitochondrial inner membrane potential $(\Delta \Psi \mathrm{m})$ to assess the protective role of Pim-1 in regulating mitochondriamediated apoptosis in MSCs based on TMRE assay. Maintenance of $\triangle \Psi \mathrm{m}$ in MSCs, MSCz, and MSCzP under normal conditions was evidenced by TMRE fluorescence intensities above $80 \%$ (MSCs $=86 \%, \mathrm{MSCz}=80 \%$, and $\mathrm{MSCzP}=81 \%$; fig. $3 \mathrm{a}$ ) throughout the 20 -min time course. MSCs and MSCz challenged with $\mathrm{H}_{2} \mathrm{O}_{2}$ showed dramatic decreases in fluorescence after $10 \mathrm{~min}$, indica- 
tive of mitochondrial inner membrane depolarization, while MSCzP maintained a fluorescent intensity comparable to that of no treatment for up to $20 \mathrm{~min}$ (fig. 3a, b). To further determine the protective effects of Pim-1 on hypoxia/SD-induced apoptosis, the assay of annexin $\mathrm{V}$ binding was performed to measure MSC apoptosis. A higher rate of cell survival was seen as evidenced by less annexin $\mathrm{V}$ binding based on a flow cytometric analysis after $24 \mathrm{~h}$ of exposure to hypoxia/SD in MSCzP compared to $\mathrm{MSCz}$ (fig. 3c). The expression kinetics of Pim$1, \mathrm{Bcl}-2$, and $\mathrm{Bcl}-\mathrm{xl}$ were also measured in MSCs, $\mathrm{MSCz}$, and MSCzP to evaluate the role of Pim-1 in the regulation of hypoxia/SD-induced apoptosis. With increasing durations of hypoxia/SD, Pim-1 and Bcl-xl levels gradually decreased. In contrast, the level of Bcl-2 was seldom influenced initially and increased gradually after $48 \mathrm{~h}$ (fig. 3d).

\section{Phenotype and Multipotential Were Maintained in $M S C z P$}

To determine the effects of elevated Pim-1 activity on the MSC phenotype and the multilineage differentiation potential, cell surface markers, cell morphology, and the trilineage differentiation potential were analyzed in MSCs, MSCz, and MSCzP using flow cytometry, inverted microscopy, and standard differentiation protocols, respectively. MSCzP uniformly showed a high level of expression of the hyaluronate receptor CD44 and the activated leukocyte cell adhesion molecule CD166, lacked expression of the leukocyte marker CD45 and the endothelial marker CD31 (as did MSCs and MSCz), and retained undifferential percentages of positive cells from those of MSCs and MSCz (fig. 4a). Consistently, MSCzP maintained the typical morphology of MSCs, with a spindle-shaped, fibroblastic morphology similar to that of MSCs and MSCz in culture (fig. 4b). Differentiation of MSCs, MSCz, and MSCzP demonstrated that MSCzP maintained multipotential differentiation towards adipogenesis, osteogenesis, and chondrogenesis (fig. 4c). These data demonstrated that Pim-1 has little or no effect on the phenotype and differentiation potential of propagated ovine MSCs.

\section{Discussion}

Ovine models are broadly used in large animal studies prior to clinical studies in a variety of fields including heart and lung ailments. Further understanding of ovine MSCs and those transduced with Pim-1 may provide im- proved ovine models for cellular therapy development. Previous studies have demonstrated that Pim-1 can promote DNA replication under normal culture conditions in rat MSCs [Tu et al., 2011]. Here, we further investigated the effects of elevated Pim-1 activity on MSC growth, survival, and differentiation using genetically modified ovine MSCs that overexpressed Pim-1. Our findings include: (1) Pim-1 enhanced intrinsic molecular signals of growth and survival implicated in the mediation of exogenous serum signaling, (2) Pim-1 promoted MSC proliferation irrespectively of serum concentrations but with decreased proliferation rates over increased serum concentrations relative to the vector-only control MSCz, (3) Pim-1 reduced the MSC apoptosis induced by hypoxia/SD, and (4) the phenotype and multipotential differentiation were maintained in Pim-1 overexpressing MSCzP.

The ERK/MEK/Raf pathway is one of the key cascades that mediate exogenous serum signaling [Gineitis and Treisman, 2001]. It consists of a chain of proteins that can communicate a signal from a receptor on the surface of the cell to the DNA in the nucleus, and ERK and Raf are the two central components [Gineitis and Treisman, 2001; Peyssonnaux and Eychène, 2001]. The ERK/MEK/ Raf pathway plays an important role in regulating cell proliferation and protection against apoptosis, and the activity of the pathway is itself dependent on phosphorylation of the components such as ERK and Raf [Gineitis and Treisman, 2001]. The elevated expression of phosphorylated ERK and Raf in our study indicated that Pim1 enhanced the activity of the ERK/MEK/Raf pathway, thus resulting in the signal of promotion of cell proliferation and antiapoptosis. The Bcl-2 family members Bcl-2 and $\mathrm{Bcl}-\mathrm{xl}$ are the key regulators of the mitochondriamediated pathway [Borillo et al., 2010]. Downregulation of Bcl-xl has been reported to be associated with increased sensitivity to apoptosis and the ectopic expression of Bcl2 -induced apoptosis in undifferentiated human MSCs [Oliver et al., 2011]. Upregulation of Bcl-xl and downregulation of Bcl-2 by Pim-1 might be indicative of enhanced regulation signals against apoptosis in MSCs. Additionally, Bcl-2 has been demonstrated to have an inhibitory effect on cell proliferation by antagonizing cell cycle regulation [Cheng et al., 2004], and thus a decreased expression of Bcl-2 might favor MSC proliferation. Overexpression of c-myc has been shown to increase cell sensitivity to apoptosis induction [Arango et al., 2003], and therefore stable expression of c-myc is, in a sense, beneficial for cellular protection against apoptosis in MSCs. Unexpectedly, the expression of p27 ${ }^{\mathrm{Kip} 1}$, a cell cycle inhibitor of checkpoint kinase cyclin-dependent kinase 2 
(cdk2)/cyclin E, and blocking of cell cycle progression through G1/S transition increased in MSCzP regardless of downregulation by Pim-1 in cardiac progenitor cells [Fischer et al., 2009], which might be indicative of inhibitory regulation on MSC propagation. However, other than to regulate MSC proliferation, $\mathrm{p} 27^{\mathrm{Kip} 1}$ participates in the regulation of cell apoptosis by inhibiting the activity of cyclin A-associated cdk2, whose upregulation is associated with cell apoptosis [Levkau et al., 1998], and therefore the upregulated $\mathrm{p} 27^{\mathrm{Kip} 1}$ expression might be indicative of enhanced antiapoptosis. Collectively, these results show that elevated Pim-1 activity enhances the intrinsic molecular signals of growth and survival implicated in mediation of serum signaling in MSCs.

In line with its role in the enhancement of mitogenic signaling, Pim-1 can promote ovine MSC proliferation under normal culture conditions, as it does in other dividing cells [Katakami et al., 2004; Aksoy et al., 2007], and in the condition of lower serum concentrations (fig. 2a). However, the promotion of cell proliferation decreases with increased serum concentrations, as evidenced by the decrease in the relative proliferation rate with an increase in serum concentration in the medium (fig. 2b). On the other hand, serum is indispensable for the promotion of cell proliferation of Pim-1, as demonstrated by the findings that MSCzP showed an 11.8-fold increase in proliferation at $5 \%$ serum and a 12.8 -fold increase at $10 \%$ serum while only displaying a 4.9 -fold increase at $1 \%$ serum relative to the initial cell number during a 7 -day culture.

Moreover, inhibition of hypoxia/SD-induced apoptosis by Pim-1 was demonstrated as evidenced by enhanced protection of mitochondrial inner membrane depolarization and reduced annexin V binding in our study. MSCs in propagation are very sensitive to hypoxia/SD and tend to be apoptotic mainly through mitochondria-mediated pathways [Zhu et al., 2006]. Depolarization of the mitochondria inner membrane resulting in alteration of the inner membrane potential is one of most important events related to cell apoptosis [Borillo et al., 2010]. Preservation of the mitochondria membrane potential in our study indicated that Pim-1 could preserve the integrity of mitochondria and therefore promote the resistance of mitochondria to apoptosis in ovine MSCs. Furthermore, the reduced annexin $\mathrm{V}$ also indicated that Pim-1 reduced the MSC apoptosis induced by hypoxia/SD. However, it should be pointed out that, as was the limitation of Pim-1 in regulating MSC proliferation, the protective effect of Pim-1 on MSC apoptosis was weakened with the duration of hypoxia/SD as demonstrated by the decreased expression of Pim-1 and prosurvival protein Bcl-xl and the in- creased expression of proapoptotic protein $\mathrm{Bcl}-2$. Collectively, our results showed that elevated Pim-1 activity regulated the intrinsic apoptosis machinery and enhanced antiapoptotic actions in MSCs.

Maintenance of the stem cell phenotype and multipotential differentiation is critically important for expanding MSCs. Propagation in cell culture can efficiently maintain MSC characteristics for several generations. Although studies have shown that Pim-1 plays a pivotal role in endothelial cell differentiation in cardiac Sca-1+ cells [Iwakura et al., 2011], our results indicated that Pim-1 has little or no effect on the phenotype and the expected multipotential differentiation of MSCs as evidenced by the following findings in MSCzP: (1) cells maintained the typical morphology of MSCs, with a spindle-shaped, fibroblastic morphology similar to that of MSCs and MSCz in culture; (2) MSCzP uniformly displayed a high level of expression of the hyaluronate receptor CD44 and the activated leukocyte cell adhesion molecule CD166 and lacked expression of the leukocyte marker CD45 and the endothelial marker CD31, and the percentages of positive cells were the same as in MSC and MSCz, and (3) multipotential differentiation towards adipogenesis, osteogenesis, and chondrogenesis was maintained. These results, together with the promotion of cell proliferation and antiapoptosis, demonstrate the supportive role of Pim-1 in regulating MSC propagation.

In conclusion, we demonstrated that elevated Pim-1 activity cooperated with exogenous serum signaling to promote ovine MSC proliferation and inhibit cell apoptosis and played a role in supporting MSC propagation in cell culture systems. Pim-1 can be activated by multiple growth factors, cytokines, and mechanics, and therefore it may participate in the regulation of multiple biological processes involving MSC propagation. Our findings, together with the results previously reported by Quijada et al. [2012], reveal that Pim-1 cooperates with serum to support MSC proliferation and survival.

\section{Acknowledgment}

We thank Dr. Paul Wilder for plate reader support, and Dr. Lili Wang (National Institute of Standards and Technology) for cellsorting support. This work was partially funded by the National Institutes of Health (grant No. R01HL 081106 to Dr. Bartley P. Griffith) and the William G. McGowan Charitable Fund. 


\section{References}

Aho, T.L., J. Sandholm, K.J. Peltola, H.P.Mankonen, M. Lilly, P.J. Koskinen (2004) Pim-1 kinase promotes inactivation of the pro-apoptotic Bad protein by phosphorylating it on the Ser112 gatekeeper site. FEBS Lett 571: 43-49.

Aksoy, I., C. Sakabedoyan, P.Y. Bourillot, A.B. Malashicheva, J. Mancip, K. Knoblauch, M. Afanassieff, P. Savatier (2007) Self-renewal of murine embryonic stem cells is supported by the serine/threonine kinases Pim-1 and Pim3. Stem Cells 25: 2996-3004.

Arango, D., J.M. Mariadason, A.J. Wilson, W. Yang, G.A. Corner, C. Nicholas, M.J. Aranes, L.H. Augenlicht (2003) c-Myc overexpression sensitises colon cancer cells to camptothecininduced apoptosis. Br J Cancer 89: 17571765.

Bachmann, M., T. Moroy (2005) The serine/threonine kinase Pim-1. Int J Biochem Cell Biol 37: 726-730.

Borillo, G.A., M. Mason, P. Quijada, M. Völkers, C. Cottage, M. McGregor, S. Din, K. Fischer, N. Gude, D. Avitabile, S. Barlow, R. Alvarez, S. Truffa, R. Whittaker, M.S. Glassy, A.B. Gustafsson, S. Miyamoto, C.C. Glembotski, R.A. Gottlieb, J.H. Brown, M.A. Sussman (2010) Pim-1 kinase protects mitochondrial integrity in cardiomyocytes. Circ Res 106: 1265-1274.

Cheng, N., Y.M. Janumyan, L. Didion, C. Van Hofwegen, E. Yang, C.M. Knudson (2004) $\mathrm{Bcl}-2$ inhibition of T-cell proliferation is related to prolonged T-cell survival. Oncogene 23: $3770-3780$

Cottage, C.T., B. Bailey, K.M. Fischer, D. Avitable, B. Collins, S. Tuck, P. Quijada, N. Gude, R. Alvarez, J. Muraski, M.A. Sussman (2010) Cardiac progenitor cell cycling stimulated by Pim-1 kinase. Circ Res 106: 891-901.

Deng, J., Y. Han, C. Yan, X. Tian, J. Tao, J. Kang, S. Li (2010) Overexpressing cellular repressor of E1A-stimulated genes protects mesenchymal stem cells against hypoxia- and serum deprivation-induced apoptosis by activation of PI3K/Akt. Apoptosis 15: 463-473.

Fischer, K.M., C.T. Cottage, W. Wu, S. Din, N.A. Gude, D. Avitabile, P. Quijada, B.L. Collins, J. Fransioli, M.A. Sussman (2009) Enhancement of myocardial regeneration through genetic engineering of cardiac progenitor cells expressing Pim-1 kinase. Circulation 120: 2077-2087.
Gineitis, D., R. Treisman (2001) Differential usage of signal transduction pathways defines two types of serum response factor target gene. JBC 276: 24531-24539.

Gnecchi, M., Z. Zhang, A. Ni, V.J. Dzau (2008) Paracrine mechanisms in adult stem cell signaling and therapy. Circ Res 103: 1204-1219.

Iwakura, T., T. Mohri, T. Hamatani, M. Obana, T. Yamashita, M. Maeda, N. Katakami, H. Kaneto, T. Oka, I. Komuro, J. Azuma, H. Nakayama, Y. Fujio (2011) STAT3/Pim-1 signaling pathway plays a crucial role in endothelial differentiation of cardiac resident Sca- $1^{+}$cells both in vitro and in vivo. J Mol Cell Cardiol 51: 207-214.

Katakami, N., H. Kaneto, H. Hao, Y. Umayahara, Y. Fujitani, K. Sakamoto, S. Gorogawa, T. Yasuda, D. Kawamori, Y. Kajimoto, M. Matsuhisa, C. Yutani, M. Hori, Y. Yamasaki (2004) Role of pim-1 in smooth muscle cell proliferation. J Biol Chem 279: 54742-54749.

Levkau, B., H. Koyama, E.W. Raines, B.E. Clurman, B. Herren, K. Orth, J.M. Roberts, R. Ross (1998) Cleavage of p21Cip1/Waf1 and p27Kip1 mediates apoptosis in endothelial cells through activation of Cdk2: role of a caspase cascade. Mol Cell 1: 553-563.

Muraski, J.A., M. Rota, Y. Misao, J. Fransioli, C. Cottage, N. Gude, G. Esposito, F. Delucchi, M. Arcarese, R. Alvarez, S. Siddiqi, G.N. Emmanuel, W. Wu, K. Fischer, J.J. Martindale, C.C. Glembotski, A. Leri, J. Kajstura, N. Magnuson, A. Berns, R.M. Beretta, S.R. Houser, E.M. Schaefer, P. Anversa, M.A. Sussman (2007) Pim-1 regulates cardiomyocyte survival downstream of Akt. Nat Med 13: 14671475.

Oliver, L., E. Hue, J. Rossignol, G. Bougras, P. Hulin, P. Naveilhan, D. Heymann, L. Lescaudron, F.M. Vallette (2011) Distinct roles of $\mathrm{Bcl}-2$ and $\mathrm{Bcl}-\mathrm{Xl}$ in the apoptosis of human bone marrow mesenchymal stem cells during differentiation. PLoS One 6: e19820.

Peyssonnaux, C., A. Eychène (2001) The Raf/ MEK/ERK pathway: new concepts of activation. Biol Cell 93: 53-62.
Pittenger, M.F., B.J. Martin (2004) Mesenchymal stem cells and their potential as cardiac therapeutics. Circ Res 95: 9-20.

Quijada, P., H. Toko, K.M. Fischer, B. Bailey, P. Reilly, K.D. Hunt, N.A. Gude, D. Avitabile, M.A. Sussman (2012) Preservation of myocardial structure is enhanced by Pim-1 engineering of bone marrow cells, Circ Res 111: 77-86.

Spiropoulos, A., M. Theodosaki, K. Stefanaki, G. Paterakis, M. Tzetis, K. Giannikou, E. Petrakou, M.N. Dimopoulou, I. Papassotiriou, E.S. Roma, E. Kanavakis, S. Graphakos, E. Goussetis (2011) Rapid clinical-scale propagation of mesenchymal stem cells using cultures initiated with immunoselected bone marrow CD105 ${ }^{+}$cells. J Cell Mol Med 15: 1983-1988.

Tu, M.L., H.Q. Wang, X.D. Sun, L.J. Chen, X.C. Peng, Y.H. Yuan, R.M. Li, X.Z. Ruan, D.S. Li, Y.J. Xu, Z.J. Ke (2011) Pim-1 is up-regulated by shear stress and is involved in shear stressinduced proliferation of rat mesenchymal stem cells. Life Sci 88: 233-238.

Wang, Z., N. Bhattacharya, M. Weaver, K. Petersen, M. Meyer, L. Gapter, N.S. Magnuson (2001) Pim-1: a serine/threonine kinase with a role in cell survival, proliferation, differentiation and tumorigenesis. J Vet Sci 2: 167179

Williams, A.R., J.M. Hare (2011) Mesenchymal stem cells: biology, pathophysiology, translational findings, and therapeutic implications for cardiac disease. Circ Res 109: 923-940.

Yankey, G.K., T. Li, A. Kilic, G. Cheng, A. Satpute, K. Savai, S. Li, S.L. Moainie, D. Prastein, C. DeFillipi, Z.J. Wu, B.P. Griffith (2008) Regional remodeling strain and its association with myocardial apoptosis after myocardial infarction in an ovine model. J Thorac Cardiovasc Surg 135: 991-998.

Zhao, Y.S., T. Li, X. Wei, G. Bianchi, J. Hu, P.G. Sanchez, K. Xu, P. Zhang, M.F. Pittenger, Z.J. Wu, B.P. Griffith (2012) Mesenchymal stem cell transplantation improves regional cardiac remodeling following ovine infarction. Stem Cells Transl Med 1: 685-695.

Zhu, W., J. Chen, X. Cong, S. Hu, X. Chen (2006) Hypoxia and serum deprivation-induced apoptosis in mesenchymal stem cells. Stem Cells 24: 416-425. 